\title{
Surgical management of carcinomas of the infratemporal fossa and skull base: patterns of failure and predictors of long-term outcomes
}

\author{
Moran Amit, MD, PhD, ${ }^{1}$ Diana Bell, MD, ${ }^{2}$ Patrick J. Hunt, BS, ${ }^{3,4}$ Ehab Hanna, MD, ${ }^{1}$ \\ Shirley Y. Su, MBBS, ${ }^{1}$ Michael Kupferman, MD, ${ }^{1}$ Mohamed Aashiq, MBBS, ${ }^{1}$ \\ Hideaki Takahashi, MD, PhD, ${ }^{1}$ Paul W. Gidley, MD, ${ }^{1}$ Marc-Elie Nader, MD, ${ }^{1}$ \\ Franco DeMonte, MD, ${ }^{4}$ and Shaan M. Raza, MD ${ }^{4}$
} 1Department of Head and Neck Surgery, Division of Surgery; ${ }^{2}$ Department of Pathology; ${ }^{3}$ Baylor College of Medicine; and
4Department of Neurosurgery, Division of Surgery, The University of Texas MD Anderson Cancer Center, Houston, Texas

\begin{abstract}
OBJECTIVE Infratemporal fossa (ITF) tumors are unique in histological characteristics and difficult to treat. Predictors of patient outcomes in this context are not known. The objective of this study was to identify independent predictors of outcome and to characterize patterns of failure in patients with ITF carcinoma.
\end{abstract}

METHODS All patients who had been surgically treated for anterolateral skull base malignancy between 1999 and 2017 at the authors' institution were retrospectively reviewed. Patient demographics, preoperative performance status, tumor stage, tumor characteristics, treatment modalities, and pathological data were collected. Primary outcomes were disease-specific survival (DSS) and local progression-free survival (LPFS) rates. Overall survival (OS) and patterns of progression were secondary outcomes.

RESULTS Forty ITF malignancies with skull base involvement were classified as carcinoma. Negative margins were achieved in 23 patients (58\%). Median DSS and LPFS were 32 and 12 months, respectively. Five-year DSS and OS rates were $55 \%$ and $36 \%$, respectively. The 5 -year LPFS rate was $69 \%$. The 5 -year overall PFS rate was $53 \%$. Disease recurrence was noted in $28 \%$ of patients. Age, preoperative performance status, and margin status were statistically significant prognostic factors for DSS. Lower preoperative performance status and positive surgical margins increased the probability of local recurrence.

CONCLUSIONS The ability to achieve negative margins was significantly associated with improved tumor control rates and DSS. Cranial base surgical approaches must be considered in multimodal treatment regimens for anterolateral skull base carcinomas.

https://thejns.org/doi/abs/10.3171/2020.3.JNS192630

KEYWORDS infratemporal fossa; anterolateral skull base; surgery; carcinoma; cancer; recurrence; survival; oncology; skull base

A NTEROLATERAL skull base malignancies with infratemporal fossa (ITF) involvement comprise a unique set of tumors with surgical considerations and histological diagnoses distinct from those for tumors of the central anterior skull base and temporal bone malignancies. The surgical challenges unique to the anterolateral skull base have been demonstrated in several key articles showing higher complication rates and reduced tumor control and survival rates. ${ }^{1}$ The surgical challenges relate to the complexity of the ITF, its adjacent skull base, and intracranial structures. The ITF is bound by the maxillary sinus anteriorly, the mandible laterally, the pterygoid plates medially, the stylopharyngeal fascia/parapharyngeal space posteriorly, and the middle cranial fossa superiorly. ${ }^{2-7}$ There are numerous channels through which disease can extend into adjacent anatomical compartments via direct spread or perineural invasion.

Numerous surgical approaches have been described for the management of tumors involving this region of the skull base, including the Fisch C, subtemporal fossa-infratemporal fossa (STF-ITF), and maxillary swing approaches. ${ }^{3,4,7-10}$ More recently, endoscopic approaches that

ABBREVIATIONS CN = cranial nerve; DSS = disease-specific survival; ITF = infratemporal fossa; KPS = Karnofsky Performance Status; LPFS = local progression-free survival; OS = overall survival; STF-ITF = subtemporal fossa-ITF.

SUBMITTED September 23, 2019. ACCEPTED March 31, 2020.

INCLUDE WHEN CITING Published online June 12, 2020; DOI: 10.3171/2020.3.JNS192630. 
use a transpterygoid or Denker's modification have also been proposed in the management of ITF pathology. ${ }^{8,11}$ The outcomes reported in most surgical series have been based primarily on the management of benign pathology. As new surgical strategies are developed, there is a need for a baseline understanding of the role that surgery plays in the management of anterolateral skull base malignancies and the factors that affect long-term outcomes.

Although numerous publications have reported surgical techniques, relatively few have reported disease-related outcomes in the management of cancers of the anterolateral skull base and ITF. ${ }^{1,3,4,9,10}$ However, anatomical and surgical considerations in the ITF are unique. Hence, outcome analysis focused on surgically treated patients with ITF malignancies is crucial. Furthermore, most of the publications on ITF malignancies have described mixed cohorts (benign and malignant), and in those that exclude benign lesions, most patients have a diagnosis of sarcoma. A previous study from our institution reporting surgical outcomes for malignant anterolateral skull base pathology demonstrated the impact of histological category (carcinoma vs sarcoma) on outcomes. ${ }^{4}$ While sarcomas require a unique multimodal approach that almost homogeneously requires chemotherapy, this is not the case with epithelial malignancies. To help shed light on the surgical and management challenges unique to carcinomas involving the ITF, we performed a retrospective review of our institutional experience; specifically, we focused on the effect of multicompartmental skull base resections and the factors that affect long-term tumor control and survival.

\section{Methods}

The study was approved by our institutional review board, which waived the requirement for informed consent. After obtaining board approval, we performed a retrospective review of the prospectively collected Brain and Spine Tumor Registry for all patients who had been surgically treated for anterolateral [skull] base malignancy between January 1999 and December 2017. This review was performed in compliance with institutional regulations with regard to the study of human subjects.

Patient imaging was reviewed to confirm involvement of the ITF and skull base. Patient demographics (age, sex, ethnicity, and diagnosis date), preoperative performance status (Karnofsky Performance Status [KPS]), tumor stage, tumor characteristics, treatment modalities, pathological data (diagnosis, grade, margin status, perineural invasion, and local invasion), and disease status were collected. In all cases, the pathological slides were rereviewed by a head and neck pathologist at our institution. All cases were presented at a multidisciplinary conference. Tumor extent was evaluated clinically and radiographically; a minority of patients with well-confined disease were treated with surgery only. This may be because of the limited nature of the disease or the preference of the treating clinician. In contrast, in patients with less confined, resectable disease, a surgical approach aimed at achieving gross-total resection is favored. Adjuvant radiation therapy is offered to all patients in whom contraindications such as previous radiation do not exist. Neoadjuvant chemotherapy is offered to patients with squamous cell and salivary gland carcinomas that are believed to be borderline resectable, while adjuvant chemotherapy is offered for all such histologies. For basal cell carcinomas, chemotherapy is not currently considered as part of the treatment paradigm for advanced surgical disease. Eight to 12 weeks after completion of definitive treatment, all patients were evaluated for disease progression by cross-sectional imaging (CT or MRI).

The primary outcome measures were disease-specific survival (DSS) and local progression-free survival (LPFS) rates. Overall survival (OS) and patterns of progression were assessed as secondary outcomes. The index date was set as the 1st day of definitive treatment at our institution; in 3 cases, previously irradiated lesions did not have a second course after surgery at our institution. For these cases, all time-to-event analyses were adjusted for prior radiation therapy. Recurrence was defined as any radiographic evidence of disease following the completion of initial treatment. LPFS was defined as radiographic evidence of progression within the skull base, head and neck, or intracranial cavities. Disease-specific survival was defined as death related to disease-specific causes.

\section{Statistical Analysis}

Continuous variables were summarized with means and medians, whereas categorical variables were summarized with frequencies and percentages. Summary statistics were calculated for all variables. The DSS and LPFS were analyzed by the Kaplan-Meier method and compared using the log-rank test. The Cox proportional hazards model was used in the univariate and multivariate analyses to identify factors that were predictive of DSS and LPFS. All statistical testing was two-tailed. Alpha was set to 0.05 for significance. All statistical testing was completed with the use of SAS JMP Pro software version 12.1.0 (SAS Institute Inc.) and R version 3.1.3 (R Foundation for Statistical Computing, open source, www.r-project.org).

\section{Results}

We identified 40 ITF carcinomas with skull base involvement. The baseline clinical and pathological characteristics of the 40 patients are listed in Table 1. Thirty patients $(75 \%)$ were men, and $10(25 \%)$ were women. The median age at treatment initiation was 60 years (range $31-81$ years). Twenty-four patients $(60 \%)$ presented to our institution after undergoing treatment elsewhere. Among those patients, 18 (45\%) had undergone prior surgery, and $12(30 \%)$ had received prior radiation therapy; 9 of the latter 12 had received concurrent chemotherapy.

Two-thirds of the previously treated patients (16 [67\%] of 24) presented with measurable disease within 6 months of having completed their prior treatment. The most common diagnosis was squamous cell carcinoma (25 patients [63\%]), followed by salivary gland malignancies (10 patients $[25 \%])$.

\section{Tumor Extent and Treatment Strategies}

Negative margins were surgically achieved in 23 patients (58\%), whereas positive microscopic margins remained in 17 patients (43\%). The anatomical extent of tu- 
TABLE 1. Baseline clinicopathological data in 40 patients with ITF tumors having skull base involvement

\begin{tabular}{|c|c|c|}
\hline Variable & No. & $\%$ \\
\hline Median age in yrs (range) & $60(31-81)$ & 100 \\
\hline \multicolumn{3}{|l|}{ Sex } \\
\hline Male & 30 & 75 \\
\hline Female & 10 & 25 \\
\hline \multicolumn{3}{|l|}{ Performance status: KPS } \\
\hline 50 & 1 & 3 \\
\hline 60 & 1 & 3 \\
\hline 70 & 7 & 18 \\
\hline 80 & 12 & 30 \\
\hline 90 & 15 & 38 \\
\hline 100 & 4 & 10 \\
\hline \multicolumn{3}{|l|}{ Prior surgery } \\
\hline Yes & 18 & 45 \\
\hline No & 22 & 55 \\
\hline \multicolumn{3}{|l|}{ Prior RT } \\
\hline Yes & 12 & 30 \\
\hline No & 28 & 70 \\
\hline \multicolumn{3}{|l|}{ Overall treatment } \\
\hline Surgery & 8 & 20 \\
\hline Surgery + RT & 13 & 33 \\
\hline Surgery + chemo-RT & 19 & 48 \\
\hline Mean FU in mos (SD) & $43(7.93)$ & 100 \\
\hline
\end{tabular}

$\mathrm{FU}=$ follow-up; $\mathrm{RT}$ = radiation therapy.

mors in the cohort, segregated by margin status, is shown in Table 2. Histopathological examination revealed that 22 patients $(55 \%)$ had local invasion, defined as invasion of the adjacent structures (bone, dura, brain, or cavernous sinus). Perineural invasion was identified in 19 patients (48\%), with cranial nerve $(\mathrm{CN}) \mathrm{V} 2$ most commonly involved (11 patients [28\%]), followed by CN V3 (10 patients [25\%]) and CN VII (20 patients [50\%]). The orbit was involved in 17 patients (43\%) with tumors extending to the ITF, as compared with no patients with tumors originating from the ITF ( $\mathrm{p}=0.003)$. Analysis of the association between invasion into different anatomical sites (i.e., invasion into individual sinuses, $\mathrm{CNs}$, intercompartmental barriers such as dura and bone) and margin status revealed that the risk for a pathological positive margin status was twofold (59\%) in patients with maxillary sinus involvement compared to those who had no involvement of the maxillary sinus (33\%); however, we found no heterogeneity between the 6 subcranial compartments (nasal cavity, orbit, and maxillary, sphenoid, frontal, and ethmoid sinuses; $\mathrm{p}=0.759$ ). Involvement of other sinuses, bone, dura, or $\mathrm{CN}$ invasion was not associated with the final margin status. Fourteen patients had postoperative complications, most commonly $\mathrm{CN}$ injury $(\mathrm{n}=4)$ and cerebrospinal fluid leakage $(\mathrm{n}=4)$, followed by respiratory failure $(n=2)$. The complication rates in patients with positive margins was 59\% compared to $17 \%$ in the patients with negative margins $(\mathrm{p}=0.012)$.

Surgical strategies were tailored to the epicenter and
TABLE 2. Comparison of resection margins according to anatomical extension

\begin{tabular}{|c|c|c|c|}
\hline Variable & $\begin{array}{l}\text { GTR w/ } \\
\text { Positive } \\
\text { Margins }\end{array}$ & $\begin{array}{l}\text { GTR w/ } \\
\text { Negative } \\
\text { Margins }\end{array}$ & $\begin{array}{c}p \\
\text { Value }\end{array}$ \\
\hline No. of patients & 17 & 23 & \\
\hline \multicolumn{4}{|l|}{ Intracranial extension } \\
\hline Bony skull base & $6(35 \%)$ & $6(26 \%)$ & 0.728 \\
\hline Dural involvement & $8(47 \%)$ & $8(35 \%)$ & 0.521 \\
\hline Brain invasion & $0(0 \%)$ & $3(13 \%)$ & 0.248 \\
\hline Cavernous sinus involvement & $3(18 \%)$ & $6(26 \%)$ & 0.707 \\
\hline Subcranial compartment & & & $0.759^{*}$ \\
\hline Nasal cavity & $5(29 \%)$ & $6(26 \%)$ & \\
\hline Frontal sinus & $2(12 \%)$ & $2(4 \%)$ & \\
\hline Ethmoid sinus & $7(41 \%)$ & $4(9 \%)$ & \\
\hline Maxillary sinus & $10(59 \%)$ & $5(22 \%)$ & \\
\hline Orbit & $9(53 \%)$ & $8(35 \%)$ & \\
\hline Sphenoid sinus & $3(18 \%)$ & $5(22 \%)$ & \\
\hline \multicolumn{4}{|l|}{ Cranial nerve involvement } \\
\hline $\mathrm{CN} \|$ & $2(12 \%)$ & $1(4 \%)$ & 0.432 \\
\hline $\mathrm{CNI}$ & 0 & $1(4 \%)$ & NA \\
\hline CN III & 0 & 0 & NA \\
\hline CN IV & 0 & 0 & NA \\
\hline CN V1 & 0 & 0 & NA \\
\hline CN V2 & $5(29 \%)$ & $6(26 \%)$ & 0.971 \\
\hline CN V3 & $4(24 \%)$ & $6(26 \%)$ & 0.712 \\
\hline CN VI & $1(6 \%)$ & $1(5 \%)$ & 0.884 \\
\hline CN VII & $9(53 \%)$ & $11(48 \%)$ & 0.980 \\
\hline CN VIII & $3(18 \%)$ & $2(9 \%)$ & 0.471 \\
\hline CN IX & 0 & $2(9 \%)$ & 0.115 \\
\hline CNX & 0 & $2(9 \%)$ & 0.115 \\
\hline CN XI & 0 & $1(4 \%)$ & 0.270 \\
\hline CN XII & 0 & $2(9 \%)$ & 0.155 \\
\hline
\end{tabular}

GTR = gross-total resection; NA = not applicable.

${ }^{*} 6 \times 2$ chi-square table for heterogeneity between the 6 sites.

anatomical extensions of disease. Ventral transfacial surgical approaches were used in 14 patients (35\%), and lateral approaches incorporating the STF-ITF approach were used in 26 patients $(65 \%)$. Free tissue transfer was used for reconstruction in 38 patients (95\%). Negative margins were achieved in $5(36 \%)$ of 14 patients undergoing ventral transfacial surgical approaches compared with 17 (65\%) of the 26 undergoing the STF-ITF approach $(\mathrm{p}=0.071)$. Adjuvant radiation therapy was administered to 32 patients (80\%), and concurrent chemotherapy was administered to $19(48 \%)$. The median radiation dose delivered was 60 Gy (range 30-70 Gy). Platinum-based chemotherapy was administered to 18 patients (45\%), 13 of whom received at least one cycle of induction chemotherapy.

\section{Patterns of Progression and Predictors of DSS and LPFS}

The median DSS and LPFS for the entire cohort were 
TABLE 3. Long-term disease and survival outcomes

\begin{tabular}{cc}
\hline \multicolumn{1}{c}{ Factor } & No. of Patients $(\%)$ \\
\hline Patterns of recurrence & \\
\hline Local & $6(15)$ \\
\hline Regional & $2(5)$ \\
\hline Distant & $3(8)$ \\
\hline Vital status & \\
\hline Yes & $16(40)$ \\
\hline Dead of disease & $24(60)$ \\
\hline
\end{tabular}

32 and 12 months, respectively. The 5-year DSS and OS rates were $55 \%$ and $36 \%$, respectively. The 5-year LPFS rate was $69 \%$, while the 5 -year overall PFS rate was $53 \%$. The patterns of progression after index treatment are listed in Table 3. Disease recurrence occurred in 11 patients (28\%), 6 of whom had local recurrence; 3 , distant recurrence; and 2, regional recurrence. The 5-year DSS rates were $41 \%, 77 \%$, and $42 \%$ for patients treated with surgery, surgery plus radiation therapy, and surgery plus adjuvant chemoradiation, respectively $(\mathrm{p}=0.147)$. The 5 -year LPFS rates were $100 \%, 88 \%$, and $54 \%$ for patients treated with surgery, surgery plus radiation therapy, and surgery plus adjuvant chemoradiation, respectively $(\mathrm{p}=0.087)$.

The results of the multivariate analyses are shown in Table 4. Our regression model revealed that age, preoperative performance status, and margin status were statistically significant prognostic factors for DSS (Table 4 and
Fig. 1). For patients with a KPS $<80$, the 5 -year DSS was $3 \%$ compared with $66 \%$ in patients with a KPS of $80-100$ ( $\mathrm{p}=0.04, \log$-rank test). Patients with a negative margin status had better DSS than the patients with positive surgical margins (HR 9.16, 95\% CI 1.74-61.84, $\mathrm{p}=0.008$; Table 4). A multivariate regression model identifying factors affecting LPFS (Fig. 2) revealed that patients with a lower preoperative performance status (HR 5.81 per 10-point increment, 95\% CI 1.25-26.88, $\mathrm{p}=0.024)$ and with a positive surgical margin status (HR 3.50, 95\% CI 1.26-10.88, $\mathrm{p}=0.015$ ) had a higher probability of local recurrence.

\section{Discussion}

Our data demonstrate that, despite the numerous anatomical extensions of malignancies in this region, the ability to surgically achieve negative margins confers significant tumor control and DSS advantages. Previous reports in the literature have been contradictory with regard to the impact of margin status, likely because of the heterogeneity of histologies assessed in those series. Negative margins were successfully achieved in 55\% of our cohort, which is comparable to other reports of negative margins in ITF surgery ranging from $38 \%$ to $70 \%$. Anatomical limitations and proximity to vital structures may have limited our ability to achieve negative surgical margins. Carcinomas are considered neurotropic tumors, and given the high degree of perineural invasion, it is still unclear how extensive resections should be in cases with neural spread..$^{12-15}$ While it is well established in the management of skull base malignancies that surgery should be considered for resectable disease, this paper demonstrates the

TABLE 4. Multivariate analyses of DSS and LPFS

\begin{tabular}{|c|c|c|c|c|}
\hline \multirow[b]{2}{*}{ Factor } & \multicolumn{2}{|l|}{ DSS } & \multicolumn{2}{|l|}{ LPFS } \\
\hline & $\mathrm{HR}(95 \% \mathrm{Cl})$ & $\mathrm{p}$ Value & $\mathrm{HR}(95 \% \mathrm{Cl})$ & p Value \\
\hline Age (increase in risk for 10 yrs) & $2.69(1.21-6.19)$ & 0.004 & $1.48(0.90-2.36)$ & 0.074 \\
\hline Sex & & 0.090 & & 0.148 \\
\hline Male & 1 & & 1 & \\
\hline Female & $7.46(0.98-14.36)$ & & $2.96(0.70-19.83)$ & \\
\hline Preop performance status (per 10-point increase in KPS) & $3.86(0.84-27.98)$ & $<0.001$ & $5.81(1.25-26.88)$ & 0.024 \\
\hline Intracranial invasion & & 0.373 & & 0.144 \\
\hline Bone & 1 & & 1 & \\
\hline Dura & $0.96(0.005-2.38)$ & & $1.20(0.30-4.70)$ & \\
\hline Brain & $6.83(0.31-16.96)$ & & $2.79(0.65-13.35)$ & \\
\hline Cavernous sinus & $7.11(0.42-17.54)$ & & $3.3(0.98-12.84)$ & \\
\hline Perineural invasion & & 0.630 & & 0.639 \\
\hline Absent & 1 & & 1 & \\
\hline Present & $1.84(0.12-20.59)$ & & $1.30(0.43-4.19)$ & \\
\hline Margins status & & 0.008 & & 0.015 \\
\hline Negative & 1 & & 1 & \\
\hline Positive & 9.16 (1.74-61.84) & & $3.50(1.26-10.88)$ & \\
\hline Treatment & & 0.133 & & 0.683 \\
\hline Surgery & 1 & & 1 & \\
\hline Surgery + RT & $0.42(0.009-0.93)$ & & $0.51(0.12-2.09)$ & \\
\hline Surgery + chemo-RT & $1.08(0.0906-8.54)$ & & $1.87(0.05-40.13)$ & \\
\hline
\end{tabular}

Boldface type indicates statistical significance. 

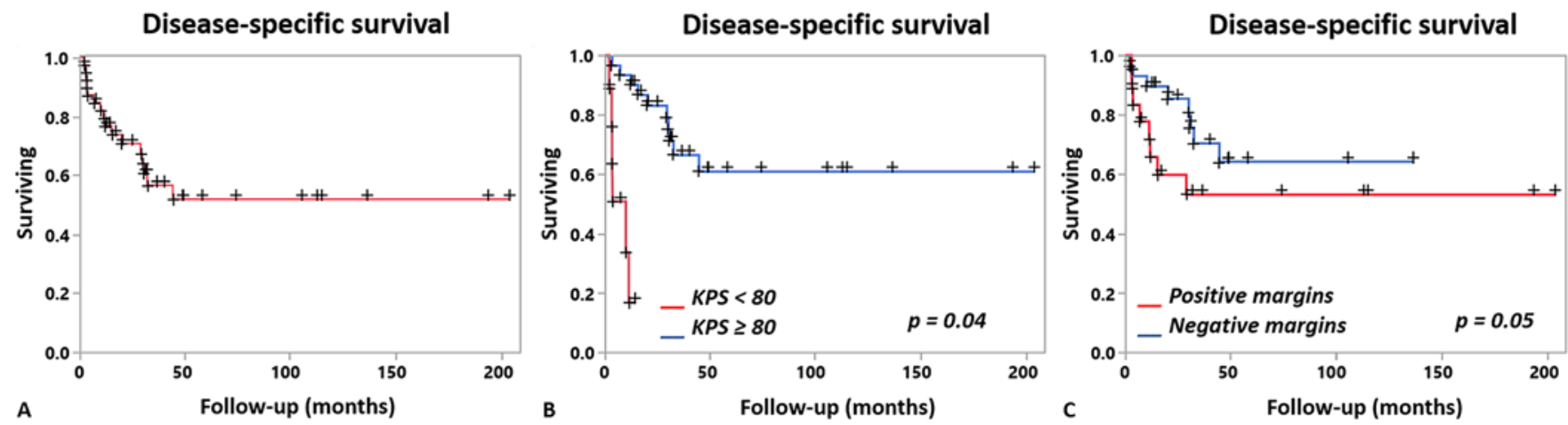

FIG. 1. Kaplan-Meier curves for DSS of overall cohort (A) and as impacted by KPS (B) and by margin status (C). The p values were calculated using the log-rank test. Figure is available in color online only.

role of surgery despite extensive perineural invasion and the availability of adjuvant therapy. ${ }^{16,17}$ This comparison is reflective of the anatomical challenges of clearing disease when the ITF and anterolateral skull base are involved.

The surgical strategies used in our series were designed to achieve negative margins in the intracranial space, along the skull base, and within the subcranial compartments. The extensive multicompartmental resections required are highlighted by the need for a free tissue transfer reconstruction in $95 \%$ of the cohort. The propensity for tumor extension into multiple anatomical compartments along with perineural invasion is evident in this study cohort. UItimately, the epicenter of disease dictated whether a ventral versus a lateral cranial base approach was used. Anatomical extensions such as dural involvement, cavernous sinus involvement, and brain invasion have been associated with a poor prognosis and prohibitive in achieving negative margins. We found that these anatomical features were not predictive of a higher risk of positive margins in our study or linked with any differences in survival or tumor control rates. ${ }^{18}$ As opposed to the anterior skull base, in which wide dural margins can be readily obtained, the anterolateral skull base requires several considerations unique to the dural anatomy. Throughout most of the anterolateral skull base, the two layers of the dura-the dura propria and the endosteal layer-are unified. The exception is along the
$\mathrm{CN}$ foramina and cavernous sinus. As the trigeminal nerve divisions pass from the intracranial to extracranial space, they are encompassed in the endosteal dural layer. Similarly, along the superior orbital fissure, the inner endosteal layer is contiguous with the periorbita. With a lateral cranial base approach, the $\mathrm{CN}$ foramina can falsely limit the degree to which margins can be obtained medially. The dura propria must be sharply dissected from the endosteal layer at the $\mathrm{CN}$ foramina in order to gain further medial access to the lateral cavernous sinus wall, which is the medial limit where dural margins can be obtained.

Within the middle cranial fossa, the venous drainage pathways limit the posterior extent to which margins can be cleared. Most importantly, the vein of Labbé ultimately enters into the dura at its distal-most drainage point into the sigmoid sinus-transverse sinus junction. Although the superior petrosal sinus can be sacrificed, the remaining venous drainage pathways in this area limit the ability to clear margins given their importance in draining the supratentorial structures. Hence, the true limits of achieving dural margins within the anterolateral skull base are the endosteal layer of the lateral cavernous sinus wall/superior orbital fissure medially and the venous drainage pathways (i.e., vein of Labbé) posteriorly.

Radiographically identified perineural invasion was present in $48 \%$ of patients but was not predictive of any
Local progression free survival

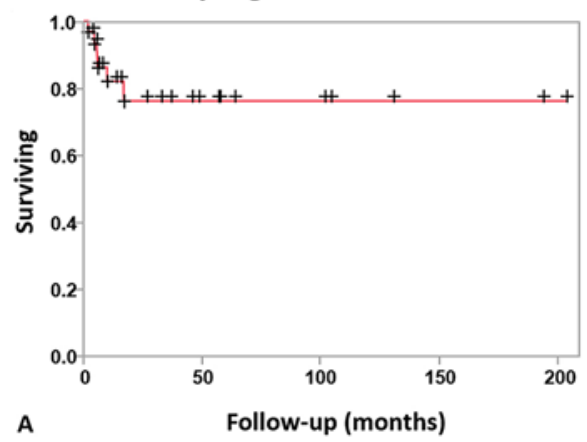

Local progression free survival

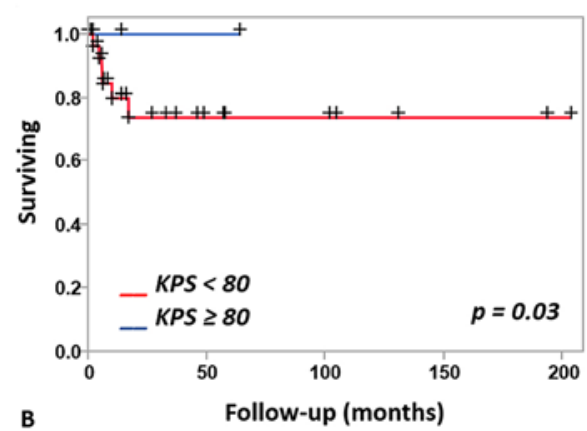

Local progression free survival

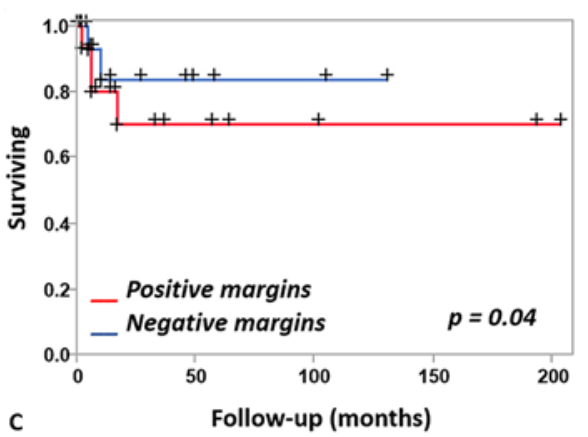

FIG. 2. Kaplan-Meier curves for LPFS of overall cohort (A) and as impacted by KPS (B) and by margin status (C). The $p$ values were calculated using the log-rank test. Figure is available in color online only. 
difference in surgical and long-term outcomes in our study. ${ }^{31,32}$ The literature has demonstrated the prognostic implications of clinical perineural invasion. In a cohort of 62 patients with cutaneous squamous cell carcinoma and basal cell carcinoma, the presence of clinical perineural invasion correlated with a significant decrease in survival rates (50\% vs $78 \%$ at 5 years). ${ }^{19,20}$ Given the technical challenges in clearing perineural invasion, several publications have proposed radiation therapy as a primary treatment modality. ${ }^{19-21}$ Conversely, numerous studies in recent years have demonstrated the positive impact of cranial base techniques with the enhanced ability to clear margins. ${ }^{15,22}$ In a cohort of patients with squamous cell carcinoma with perineural invasion, Solares et al. demonstrated the positive impact on 5-year DSS in patients undergoing curative surgery versus radiation therapy $(53.6 \%$ vs $0 \%) .{ }^{22}$ The findings from our study lend further support to the role of surgery in the management of extensive carcinomas with perineural invasion as part of a multimodal treatment paradigm.

In the anterolateral skull base, the $\mathrm{CNs}$ primarily at risk are the trigeminal nerve and facial nerve. Although the purported mechanism is retrograde invasion, anterograde spread must be considered with the various divisions of the trigeminal system. V1 spread typically occurs in conjunction with orbital invasion and is ultimately managed with an orbitectomy and a lateral cavernous sinus dissection. V2 spread requires consideration of the pterygopalatine fossa and its distal branches to the palate and along the roof of the maxillary sinus. Via a ventral approach, V2 invasion can be resected back to the gasserian ganglion by widening the foramen rotundum and removing the maxillary strut. ${ }^{23}$ With V3 involvement, careful consideration is given to the complex nerve branching within the ITF, which is typically managed with a lateral approach in which the ITF is resected. In addition, numerous communications between the facial nerve and trigeminal nerve, such as via the zygomaticotemporal nerve, could be a source of perineural extension. ${ }^{24,25}$ Lastly, the degree of facial nerve involvement typically dictates the need for a radical parotidectomy and lateral temporal bone resection. Ultimately, with regard to perineural invasion, the currently perceived anatomical limits for clearing margins for each nerve are the gasserian and geniculate ganglion, respectively. Beyond the gasserian ganglion, with involvement of the trigeminal root, there is concern for positive or close margins at the brainstem. With retrograde involvement of the facial nerve beyond the geniculate ganglion, there is concern about involvement of the greater superficial petrosal nerve (and the adventitia of the underlying carotid artery) along with the nerves within the internal auditory canal. Hence, the indications and goals of surgery for curative intent are carefully considered when radiographic involvement of these anatomical structures is present.

Although the size of our study and cohort design prevented further analysis of the effect of multimodal therapy, the treatment strategies in this population must be considered. Most of the recurrences were local; however, the presence of systemic metastases emphasized the importance of local and distant disease control. This is highlighted by the use of adjuvant radiation therapy in $80 \%$ of the cohort, with concurrent chemotherapy administered to $48 \%$ of the patients. Neoadjuvant chemotherapy was administered in $33 \%$ of the cohort. The intensive nature of the resection timed with extensive neoadjuvant and adjuvant therapies is the hypothesized explanation for the prognostic impact of performance status. ${ }^{30}$ Our study identified a KPS that was linked not only with a higher probability of local recurrence (HR 5.81 per 10-point increment, 95\% CI 1.25$26.88, \mathrm{p}=0.024)$ but also with disease-related mortality (5-year DSS $3 \%$ with KPS < 80 vs 66\% with KPS 80-100). In general, adjuvant radiation therapy is indicated for T4 malignancies and has been linked with improved survival in the histologies addressed in our study. ${ }^{26}$ Particular attention is placed on the inclusion of pathways of perineural spread in the clinical target volume. ${ }^{12}$ Because of advances in radiation therapy planning, it has become increasingly possible to spare organs at risk while delineating complex target volumes. Furthermore, in such a heavily pretreated population, in which $30 \%$ of the cohort presented for surgery after previous radiation therapy, the ability to offer repeat radiation in the salvage setting is an additional consideration. ${ }^{27-29}$

Although this is the largest series of carcinomas involving the ITF and skull base in the literature, there are limitations to this study. Given the rarity of these tumors and the small number of events for both LPFS and DSS in our cohort, the multivariate analysis is overfitted and will require multiinstitutional external validation. In addition, this was a narrow cohort of patients whose disease we had deemed resectable and to whom we offered surgical intervention. That said, other covariates (e.g., tumor invasion capacity) may have driven patient outcome; hence, we refrained from any conclusion about causality between margin status or treatment regimen and outcomes. Conclusions regarding the management of unresectable disease cannot be based on the findings of this study. Finally, innovations in chemotherapy and radiation may not be reflected in this study because of the extended study period and the types of patients selected for this study.

\section{Conclusions}

Infratemporal fossa carcinomas are anatomically extensive lesions with numerous surgical challenges relating to extensive degrees of perineural invasion and intracranial invasion that can serve as barriers to curative surgery. Our study demonstrated that resection for anterolateral skull base carcinomas in selected patients whose tumors are considered resectable can help to achieve long-term outcomes similar to those reported for carcinomas at other anatomical sites. The ability to achieve negative margins was significantly associated with improved tumor control rates and DSS. Factors such as dural invasion, cavernous sinus invasion, and perineural invasion were not prohibitive to achieving negative margins and were not associated with a poor long-term outcome. Ultimately, surgical intervention with cranial base approaches needs to be considered as a component of a multimodal treatment regimen that includes adjuvant radiation therapy and chemotherapy, the sequence of which is considered depending on the histology and extent of disease. 


\section{References}

1. Irish JC, Gullane PJ, Gentili F, et al. Tumors of the skull base: outcome and survival analysis of 77 cases. Head Neck. 1994;16(1):3-10.

2. Bejjani GK, Sullivan B, Salas-Lopez E, et al. Surgical anatomy of the infratemporal fossa: the styloid diaphragm revisited. Neurosurgery. 1998;43(4):842-853.

3. Bilsky MH, Bentz B, Vitaz T, et al. Craniofacial resection for cranial base malignancies involving the infratemporal fossa. Neurosurgery. 2005;57(4)(suppl):339-347.

4. Hentschel SJ, Vora Y, Suki D, et al. Malignant tumors of the anterolateral skull base. Neurosurgery. 2010;66(1):102-112.

5. Isolan GR, Rowe R, Al-Mefty O. Microanatomy and surgical approaches to the infratemporal fossa: an anaglyphic three-dimensional stereoscopic printing study. Skull Base. 2007;17(5):285-302.

6. Joo W, Funaki T, Yoshioka F, Rhoton AL Jr. Microsurgical anatomy of the infratemporal fossa. Clin Anat. 2013;26(4):455-469.

7. Sekhar LN, Schramm VL Jr, Jones NF. Subtemporal-preauricular infratemporal fossa approach to large lateral and posterior cranial base neoplasms. J Neurosurg. 1987;67(4):488-499.

8. Battaglia P, Turri-Zanoni M, Dallan I, et al. Endoscopic endonasal transpterygoid transmaxillary approach to the infratemporal and upper parapharyngeal tumors. Otolaryngol Head Neck Surg. 2014;150(4):696-702.

9. Givi B, Liu J, Bilsky M, et al. Outcome of resection of infratemporal fossa tumors. Head Neck. 2013;35(11):1567-1572.

10. Leonetti JP, Benscoter BJ, Marzo SJ, et al. Preauricular infratemporal fossa approach for advanced malignant parotid tumors. Laryngoscope. 2012;122(9):1949-1953.

11. van Koevering K, Prevedello DM, Carrau RL. Endoscopic endonasal approaches for the management of cranial base malignancies: histologically guided treatment and clinical outcomes. J Neurosurg Sci. 2018;62(6):667-681.

12. Armstrong K, Ward J, Hughes NM, et al. Guidelines for clinical target volume definition for perineural spread of major salivary gland cancers. Clin Oncol (R Coll Radiol). 2018;30(12):773-779.

13. Hanna EY, Cardenas AD, DeMonte F, et al. Induction chemotherapy for advanced squamous cell carcinoma of the paranasal sinuses. Arch Otolaryngol Head Neck Surg. 2011;137(1):78-81.

14. Panizza B, Solares CA, Redmond M, et al. Surgical resection for clinical perineural invasion from cutaneous squamous cell carcinoma of the head and neck. Head Neck. 2012;34(11):1622-1627.

15. Raza SM, Ramakrishna R, Weber RS, et al. Nonmelanoma cutaneous cancers involving the skull base: outcomes of aggressive multimodal management. J Neurosurg. 2015;123(3):781-788.

16. Ganly I, Patel SG, Singh B, et al. Craniofacial resection for malignant paranasal sinus tumors: report of an International Collaborative Study. Head Neck. 2005;27(7):575-584.

17. Hanna E, DeMonte F, Ibrahim S, et al. Endoscopic resection of sinonasal cancers with and without craniotomy: oncologic results. Arch Otolaryngol Head Neck Surg. 2009;135(12):1219-1224.

18. Lee YC, Lee TJ, Tsang NM, et al. Cavernous sinus involvement is not a risk factor for the primary tumor site treatment outcome of sinonasal adenoid cystic carcinoma. J Otolaryngol Head Neck Surg. 2018;47(1):12.

19. McCord MW, Mendenhall WM, Parsons JT, et al. Skin cancer of the head and neck with clinical perineural invasion. Int J Radiat Oncol Biol Phys. 2000;47(1):89-93.

20. McCord MW, Mendenhall WM, Parsons JT, Flowers FP. Skin cancer of the head and neck with incidental microscopic perineural invasion. Int J Radiat Oncol Biol Phys. 1999;43(3):591-595.
21. Jackson JE, Dickie GJ, Wiltshire KL, et al. Radiotherapy for perineural invasion in cutaneous head and neck carcinomas: toward a risk-adapted treatment approach. Head Neck. 2009;31(5):604-610.

22. Solares CA, Mason E, Panizza BJ. Surgical management of perineural spread of head and neck cancers. J Neurol Surg B Skull Base. 2016;77(2):140-149.

23. DeMonte F, Hanna E. Transmaxillary exploration of the intracranial portion of the maxillary nerve in malignant perineural disease. Technical note. J Neurosurg. 2007;107(3):672-677.

24. Amit M, Binenbaum Y, Trejo-Leider L, et al. International collaborative validation of intraneural invasion as a prognostic marker in adenoid cystic carcinoma of the head and neck. Head Neck. 2015;37(7):1038-1045.

25. Odobescu A, Williams HB, Gilardino MS. Description of a communication between the facial and zygomaticotemporal nerves. J Plast Reconstr Aesthet Surg. 2012;65(9):1188-1192.

26. Langendijk JA, Slotman BJ, van der Waal I, et al. Risk-group definition by recursive partitioning analysis of patients with squamous cell head and neck carcinoma treated with surgery and postoperative radiotherapy. Cancer. 2005;104(7):14081417.

27. Na'ara S, Amit M, Billan S, et al. Outcome of patients undergoing salvage surgery for recurrent nasopharyngeal carcinoma: a meta-analysis. Ann Surg Oncol. 2014;21(9):3056-3062.

28. Phan J, Sio TT, Nguyen TP, et al. Reirradiation of head and neck cancers with proton therapy: outcomes and analyses. Int J Radiat Oncol Biol Phys. 2016;96(1):30-41.

29. Pollard C III, Nguyen TP, Ng SP, et al. Clinical outcomes after local field conformal reirradiation of patients with retropharyngeal nodal metastasis. Head Neck. 2017;39(10):20792087.

30. Amit M, Abdelmeguid AS, Watcherporn T, et al. Induction chemotherapy response as a guide for treatment optimization in sinonasal undifferentiated carcinoma. J Clin Oncol. 2019;37(6):504-512.

31. Amit M, Eran A, Billan S, et al. Perineural spread in noncutaneous head and neck cancer: new insights into an old problem. J Neurol Surg B Skull Base. 2016;77(2):86-95.

32. Amit M, Na'ara S, Gil Z. Mechanisms of cancer dissemination along nerves. Nat Rev Cancer. 2016;16(6):399-408.

\section{Disclosures}

Dr. Nader has direct stock ownership in Johnson \& Johnson, Medtronic, Kimberly Clark, Amgen, 3M, and Pfizer.

\section{Author Contributions}

Conception and design: Raza, Amit. Acquisition of data: Raza, Amit, Bell, Hunt. Analysis and interpretation of data: Raza, Amit, Hunt. Drafting the article: Raza, Amit, Aashiq. Critically revising the article: all authors. Reviewed submitted version of manuscript: Raza, Amit, Bell, Hanna, Su, Kupferman, Aashiq, Takahashi, Gidley, Nader, DeMonte. Approved the final version of the manuscript on behalf of all authors: Raza. Statistical analysis: Raza. Study supervision: Raza.

\section{Correspondence}

Shaan M. Raza: The University of Texas MD Anderson Cancer Center, Houston, TX. smraza@mdanderson.org. 